\title{
Multi-Ensayos
}

Vol. 4, $\mathrm{N}^{\circ} 7$

ISSN: 2412-3285

https://multiensayos.unan.edu.ni

DOI: https://doi.org/10.5377/multiensayos.v4i7.9484

\section{Importancia del comportamiento organizacional para los estudiantes de Administración de Empresas}

\section{Importance of Studying Organizational Behavior for Students of Business Administration}

\author{
Alberto Sevilla Rizo ${ }^{1}$
}

Recibido: 28 de febrero de 2018, Aceptado: 27 de septiembre de 2018

\section{RESUMEN}

El estudio del comportamiento organizacional tiene una gran relevancia en el aprendizaje de los estudiantes de Administración de Empresas, dado que en esta área se trabaja con individuos y cada uno tiene diferente comportamiento, debido múltiples factores, sean estos de índole familiar, social, político, económicos, entre otros. Ello influirá en el desempeño de sus funciones de acuerdo a la percepción que cada uno de estos individuos tengan, y esto será del cúmulo de aprendizajes desde, en y para la vida. Así mismo, influirá en el trabajo de grupo que se realice dentro de la organización, lo que evitará o incidirá en los conflictos. Todo ello repercute en la eficiencia y eficacia de las funciones realizadas en el ámbito empresarial.

Palabras claves: comportamiento organizacional; individuo; percepción; conflicto; trabajo de grupo.

\section{ABSTRACT}

The study of organizational behavior has a great relevance in the process of training students of Business Administration, given that they will have to work with individuals and each one has different behavior, due to family, social, political, economic factors, among others. Such situations will influence their performance according to the perception that each of these individuals might have. In this sense, students will learn from, in and for real life. Likewise, this kind of learning will influence group work carried out within the organization, which will prevent or influence conflicts within the organization. All this impacts the efficiency and effectiveness of the functions carried out in the business sector.

Keywords: organizational behavior; individual; perception; conflict; group work.

1 Docente del Departamento de Ciencias Económicas y Administrativas, UNAN-Managua/FAREM-Estelí. Correo electrónico: asevilla_42@ yahoo.es

Copyright (c) 2018 Revista Multi-Ensayos. 


\section{INTRODUCCIÓN}

El estudio del comportamiento organizacional es sumamente importante en el aprendizaje de los estudiantes de Administración de Empresas, dado que el trabajo es principalmente relacionado con el personal. Los gerentes son aquellas personas que realizan actividades a través de otras personas dentro de una organización y esta es una entidad coordinada que busca lograr una meta o varias metas en común.

Quintero, (2003) distingue las siguientes etapas entre las actividades de un gerente: Dirección, Organización y el Control. La gerencia cumple roles muy importantes dentro de una organización; por ello es meritorio que todos los estudiantes de Administración de Empresas se apropien de los factores que se toman en cuenta en el comportamiento organizacional, para que desarrollen habilidades técnicas y apliquen conocimientos, sumado a la experiencia adquirida para desarrollar la habilidades y capacidad de trabajar con otras personas y sobrellevar situaciones complejas.

\section{DESARROLLO}

Para el desarrollo de este trabajo se tomó en cuenta el concepto del comportamiento organizacional, los factores que inciden en los colaboradores de las organizaciones, la forma de acción y la influencia que se tiene en las organizaciones. En las últimas décadas se ha venido estudiando el porqué de este comportamiento y cómo mejorarlo en busca de la eficiencia y eficacia, a fin de crear condiciones adecuadas para el desarrollo de las actividades.

De la misma manera, el propósito principal es que cada estudiante interiorice la importancia del comportamiento organizacional, ya que es con personas que tiene que intervenir y el conocimiento y la aplicación de esta temática dentro de las organizaciones se ha convertido en una parte indispensable para el buen funcionamiento de la misma, por lo que ya es un modelo para conocer y diagnosticar la funcionalidad de todos los elementos.

La organización es un sistema complejo compuesto por subsistemas físicos, sociales, técnicos, económicos y legales, que procesa un conjunto de insumos para producir bienes y servicios, en ella existen edificios, maquinarias, herramientas, instrumentos, sistemas de trabajo, flujos o procesos y personas, que al interactuar como un todo general, en busca de sus objetivos, configurara una serie de variables que afectaran sobre las conductas de las personas. (Psicologia y empresas, 2010)

Es por ello que se debe conocer todo acerca de los factores internos y externos, para una mejor dirección. Ello será de mucha utilidad por lo que los estudiantes de Administración de Empresas deben de estar claros de que el comportamiento organizacional es un campo de estudio que investiga el efecto que los individuos, grupos y estructura que tienen sobre el comportamiento dentro de las organizaciones, con el propósito de aplicar dicho conocimiento para mejorar la efectividad de las organizaciones. (Robbins \& Judge, 2009)

Para ejemplificar con un caso sencillo, pensemos en una persona que este dirigiendo una empresa, un departamento, una área; debe estar consciente de que va a trabajar con personas y que estas personas tienen 
diferentes necesidades, diferentes motivaciones, diferentes problemáticas y que por lo tanto, el objetivo y las metas de la empresa será el mismo para todo los que laboran; así pues, tendrá que dirigir y formar grupos de trabajo y aunque estos tengan diferencias, siempre deben realizar las labores encomendadas.

Robbins \& Judge( 2009) proponen que existen tres niveles de análisis en el comportamiento organizacional y que conforme se avanza del nivel del individuo al de los sistemas de la organización, aumenta en forma sistemática nuestro entendimiento del comportamiento en las organizaciones. Los tres niveles básicos son análogos a los ladrillos de construcción; cada nivel se construye sobre el anterior. Los conceptos de grupo crecen a partir del fundamento colocado en la sección del individuo; sobreponemos restricciones estructurales al individuo y al grupo a fin de llegar al comportamiento organizacional.

Este modelo lo comparten la mayoría de los estudiosos administrativos, dado que es la base fundamental para el manejo de las personas grupos y empresas; por lo que es básico estudiar cada uno de los aspectos y cada uno de los componentes de las etapas descritos en el concepto y el modelo propuesto.

De acuerdo a lo expresado en el modelo del comportamiento organizacional, los autores antes mencionados plantean que una variable dependiente es el factor clave que queremos explicar o predecir y que se ve afectada por algún otro factor. Por lo tanto, podemos estudiar las principales variables dependientes. Los estudiosos enfatizado en la productividad, ausentismo, rotación y satisfacción con el trabajo. En épocas más recientes, se han agregado a esta lista dos variables más: comportamiento que se aparta de las normas del sitio de trabajo y la ciudadanía organizacional.

Es por eso que las personas que ocupan un cargo dentro de cualquier tipo de empresa deben de saber todas estas características de la misma. De ahí que, el estudiante de Administración de Empresas está obligado a conocer y apropiarse estos términos dado que él tiene que relacionarse con este ambiente. Por eso se pude decir que el comportamiento organizacional se ocupa del estudio de lo que hacen las personas en una organización y de cómo afecta su comportamiento al desempeño de la misma.

El administrador de empresas debe de tener presente y conocer todas estas variables y así poder conocer a sus colaboradores. De acuerdo a lo anterior, Robbins \& Judge (2009) plantean que en las variables independientes se abordan las principales determinantes de la productividad, ausentismo, rotación, desviación de las normas de comportamiento en el sitio de trabajo, comportamiento organizacional y la satisfacción en el trabajo. La respuesta a tales preguntas conduce a las variables independientes. Una variable independiente es la presunta causa de cierto cambio de la variable dependiente.

En el estudio del individuo, según Robbins \& Judge (2009), los gerentes, a diferencia de los padres, deben trabajar con seres humanos usados, no nuevos seres humanos de quienes otros hicieron los primeros. Cuando un colaborador ingresa a una organización se parecen un poco a los autos usados. Así por ejemplo, una persona y otra difieren en sus características, sus creencias, motivaciones, entre otras que influyen en la forma de comportarse y de realizar las actividades. Es ahí donde radica la importancia del estudio de estas variables y que se ha vuelto fundamental, ya que de ello depende el éxito de todas las empresas; independientemente de su actividad. 
El comportamiento organizacional se refiere a los rasgos biográficos tales como la edad, género y estado civil; los rasgos de la personalidad; una estructura emocional inherente; valores, actitudes; y niveles de aptitudes básicas. En esencia, estas características ya están definidas cuando un individuo se integra a la fuerza de trabajo, y en su mayor parte hay poco que la gerencia pueda hacer para modificarlas. Aun así, tienen un efecto muy real en el comportamiento del empleado. Hay otras cuatro variables de nivel individual que se ha demostrado afectan al comportamiento de los empleados: percepción, toma individual de decisiones, aprendizaje y motivación. (Robbins \& Judge, 2009, pág. 48)

Partiendo de lo anteriormente expuesto, el estudio sobre el individuo es relevante y en los últimos años se ha tomado como referencia, ya que de la dirección depende la buena organización y los buenos resultados de la organización. Por ello es meritorio que quien dirige conozca cada una de las característica de quienes van a estar a su cargo.

Otra de las variables a tomar en cuenta son los grupos o equipo o departamento dentro de las empresas; estos grupos, están formado por individuos, algunos son bastantes comunicativos, otros apáticos; también existen otros a los que todo le incomoda, en fin, cada responsable de grupo tiene que unificar propósitos y fines de acuerdo a sus objetivos y metas propuestas; a fin de que el trabajo se realice de la mejor manera posible.

Según Robbins \& Judge (2009) el comportamiento de la gente en grupos es más que la suma de los individuos si actuaran por su cuenta. La complejidad de nuestro modelo aumenta cuando se toma en cuenta que el comportamiento de las personas cuando forman parte de grupos es diferente del que muestran cuando están solas. Por tanto, el siguiente paso en el desarrollo del entendimiento del comportamiento organizacional es el estudio del comportamiento grupal; comprensión de la dinámica del comportamiento del grupo (forma en que los individuos de los grupos reciben influencia de los patrones de comportamiento que se espera tengan, de lo que el grupo considera son estándares de comportamiento aceptable y el grado en que los miembros del grupo se atraen entre sí), entendimiento de los grupos al diseño de equipos de trabajo efectivos, y la forma en que los patrones de comunicación, liderazgo, poder y política, así como los niveles de conflicto, influyen en el comportamiento del grupo.

En esta variable la importancia radica en que los administradores deben ser mediadores y saber enfrentar conflictos, que son muy comunes cuando se trabaja con personas, esto se dará porque es algo común entre los individuos ya que cada uno de ellos tienen diferentes metas, propósitos, motivaciones, por lo que a cada uno se trata de manera diferenciada. Se debe de tener en cuenta que quien dirige determinado grupo, lo primero que tiene que hacer es conocer cada una de las características de cada uno de los individuos que lo conforman. De ahí el énfasis en que los estudiantes de Administración de Empresas están obligados a conocer e interiorizar esta temática, que será de mucha utilidad en su campo laboral.

Por otro lado, la variable de sistema de la organización tiene que ver con el hecho de que la persona que está cargo de una empresa debe conocer aspectos a nivel interno y externo, porque cada uno de ellos influirá de manera directa sobre la empresa y el papel de todo administrador es mitigar algunos aspectos que le podrían hacer daño al buen funcionamiento de la misma; e incluir o integrar aquello que será de beneficio. 
El comportamiento organizacional alcanza su nivel más alto de sofisticación cuando se agrega una estructura formal a nuestro conocimiento previo del comportamiento del individuo y el grupo. Así como los grupos son más que la suma de sus miembros, las organizaciones son más que la suma de los grupos que las forman. El diseño de la organización formal; la cultura interna de la organización y las políticas y prácticas de recursos humanos de ésta (es decir, procesos de selección, programas de capacitación y desarrollo, y métodos de evaluación del desempeño), tienen un efecto en las variables dependientes. (Robbins \& Judge, 2009)

Esta variable dependerá del buen estudio que se haga o la buena selección de cada uno de los individuos, desde la selección y contratación; esto para determinar las características para cada uno de los puestos que se requieren, y esto será una ventaja para la personas que estarán a cargo de cada uno de los departamentos en lo que se logrará un mejor desempeño del equipo de trabajo y esto beneficiará a la empresa en el logro de sus propósitos.

Para concluir se podría argumentar la importancia y la relevancia que tiene el estudio del comportamiento organizacional para los estudiantes de la carrera de Administración de Empresas, dado que explica de manera detallada cada uno de los aspectos a tomarse en cuenta para lograr con éxito el manejo del personal. Se puede tomar en cuenta que el comportamiento organizacional dependerá si se enfoca a los factores de motivación, liderazgo, así como las conductas que se presentan en el ambiente laboral. En cada uno de estos puntos se debe destacar que son de suma importancia, si se tiene una buena aplicación de estos, el ambiente laboral será agradable y motivante para los empleados y, por lo tanto, se verán los resultados en cuanto a producción por nombrar un ejemplo, y el comportamiento organizacional de una forma ayudará a los empresarios, para saber cómo actuar, o que está faltando en la organización y ambas partes tengan mejor efectividad. (Cruz Sanchez, 2016)

En suma, para alcanzar el éxito se tendrá que conocer las características, las habilidades, los conocimientos, como también las diferentes motivaciones que cada uno de los individuos tienen, esto porque el propósito, es ser eficaces y eficientes; lo que se logrará siempre y cuando se controle el comportamiento de los individuos dentro de la organización.

\section{BIBLIOGRAFÍA}

Cruz Sánchez, E. (06 de Diciembre de 2016). Factores del comportamiento Organizacional. Recuperado el 23 de febrero de 2018, de http://tustextos.com/ely/factores-del-comportamiento-organizacional/

Psicología y empresas. (19 de noviembre de 2010). Psicología y empresas. Recuperado el 23 de febrero de 2018, de https://psicologiayempresa.com/factores-que-afectan-la-contucta-en-la-organizacion. html

Quintero, T. (28 de julio de 2003). https://www.gestiopolis. Recuperado el 23 de febrero de 2018, de https://www.gestiopolis.com/el-comportamiento-organizacional/

Robbins, S., \& Judge, T. (2009). Comportamiento Organizacional. México: PEARSON Educación. 